Kinesthetic Interaction

Kyle Reed, Michael Peshkin, Mitra Hartmann, J. Edward Colgate; James L. Patton

IEEE 9th International Conference on Rehabilitation Robotics, June $28,2005,2005$

\title{
Kinesthetic Interaction
}

\author{
Kyle B. Reed, Michael Peshkin, Mitra J. Hartmann, J. Edward Colgate, and James Patton
}

\begin{abstract}
In physical and occupational therapy two people interact through force and motion. Other common examples of this interaction include lifting and moving a bulky object, teaching manual skills, dancing, and handing off a baton or a drinking glass. These tasks involve kinesthetic interaction, a communication channel distinct from spoken language and gestures. Understanding kinesthetic interaction should be important in designing robots to assist with physical and occupational therapy.

In this paper we describe our experiments on kinesthetic interaction between two people cooperating on a 1 degree of freedom task. We characterize the interaction forces between the two people, dividing them into a productive "net force" and an orthogonal "difference force." Our results suggest three effects (1) an emergent specialization of the two participants into different roles, (2) an oscillation of forces at about $8 \mathrm{~Hz}$, and (3) a steady force in opposition to one another that could be analogous to co-contraction in an individual.
\end{abstract}

\section{INTRODUCTION}

A basic form of human interaction is the physical cooperation necessary to perform a manual task with others. Physical cooperation represents a communication channel distinct from facial expression, gesture, and spoken language, yet one that has been much less studied. We expect that a significant channel of dyadic communication should be through forces and motions, applied either directly to one another's limbs or via a mutually grasped object.

Such mechanical cooperation is involved in a wide variety of tasks including moving objects too large or heavy for an individual, tasks requiring two hands for object stability while other hands perform manipulative movements, and direct physical guidance of another person's limbs for teaching and learning. Whether by direct limb-to-limb coupling or coupling through a mutually grasped object, these activities create a kinesthetic interaction between two individuals in which one person feels and reacts to the forces and motions of the other person. Cooperative mechanical control is also important for tasks whose complexity may exceed the cognitive or motor capacity of an individual, as in piloting of remote vehicles and shared control teleoperation for minimally invasive surgery.

Manuscript received February 11, 2005. This work is supported by NSF grant ECS-0433948

K. B. Reed is with the Laboratory for Intelligent Mechanical Systems, Northwestern University, Evanston, IL, 60208, USA (847-467-1070; e-mail: reedkb@northwestern.edu).

M. Peshkin is with the Laboratory for Intelligent Mechanical Systems, Northwestern University, Evanston, IL, 60208.

M. J. Hartmann is on a joint appointment with Mechanical and Biomedical Engineering, Northwestern University, Evanston, IL, 60208.

J. E. Colgate is with the Laboratory for Intelligent Mechanical Systems, Northwestern University, Evanston, IL, 60208.

J. Patton is with the Rehabilitation Institute of Chicago, IL, 60611.
Our area of interest, lower limb rehabilitation, also involves haptic interaction between two people: patient and therapist. Several previous efforts have tried to create a robot that itself performs aspects of physical therapy, such as the Lokomat [1]. In contrast to such efforts, our research strives to create a device that assists the physical therapist in physically demanding tasks. Hands-on interaction with patients is highly valued by physical therapists; we aim to enhance it, not replace it.

The hands-on interaction between physical therapist and patient involves the communication of muscle tone, force, and motion to the therapist, and the selective delivery of force and motion by the therapist. In this and further studies, we will explore the interaction between subjects to explore how two people physically cooperate, compromise, and guide one another, and how machine-generated forces and motions can enter into the human-human physical conversation.

\section{RELATED WORK}

Shared manual control occurs in learning to operate some kinds of equipment, most critically in the teaching/learning of helicopter control. Wegner \& Zeaman [2] were interested in applications of the dyadic effects they were studying, and discussed automobile and aircraft dual controls. They also identified some dual control tasks in everyday life, including the two-handled saw, the see-saw, and the balancing of a tandem bicycle. Shaw [3] adds couples dancing. Other everyday activities exhibiting dual control are maneuvering and positioning a board or other object and placing a bed sheet on a bed. In some of these examples, the participants establish a working relationship in which they must compromise (e.g. positioning the bed sheet symmetrically) or in which they must divide authority according to task phase.

Sallnas and Zhai [4] performed a study with a haptic system simulating the handoff of an object on a computer screen. They measured the time it takes for one person to hand off objects to another person (haptically simulated) within certain spatial targets. Here the two people are not physically connected, they feel only the sensations the haptic device can simulate. Similarly, two athletes pass a baton without looking and rely completely on their haptic sense to determine when the exchange is complete.

Both Elhajj et al. [5] and Hespanha et al. [6] have explored humans communicating in a teleoperational haptic environment over the internet. Barnes and Counsell [7] explored these same issues locally, much like Sallnas and Zhai's setup with the handoff computer simulation. In each of these cases, the two people were not physically or rigidly connected. 
Griffiths and Gillespie [8] demonstrated a haptic steering wheel that communicates bi-directionally with the driver. They show that this haptic steering wheel reduced the visual demand of the driver. This haptic steering wheel provides information about where the controller thinks the car should go by pushing slightly in the desired direction. The controller acts differently than a second human operator would since it is not simulating a human, which may mean that the system lacks other important haptic cues.

\section{EXPERIMENTAL APPARATUS \& PROTOCOL}

The apparatus is shown in Fig. 1. Two subjects hold the handles, one on either side of the crank. The angular position of the crank is measured by an optical encoder and displayed to each subject as a black line on the top plate of the setup. The subjects' task is to bring the dark line into a target area (shaded region of the plate in Fig. 1). The targets change color when the crank is inside the target region. The handles are connected via a rigid link that can spin freely at the center where a direct drive motor is attached (under the table). The motor was not used in the experiments reported here, but is likely to be important in future work. A projector mounted above the table (not shown) displays the targets, the motivating performance measure, and instructions to each subject. A curtain hangs between the subjects to prevent visual communication.

The targets vary between one of five sizes: $2^{\circ}(1.1 \mathrm{~cm}$ around the circle at the handle $), 4^{\circ}(2.2 \mathrm{~cm}), 7^{\circ}(3.8 \mathrm{~cm}), 13^{\circ}$ $(7.1 \mathrm{~cm})$, and $25^{\circ}(13.7 \mathrm{~cm})$ with a distance between targets of $70^{\circ} \pm 10^{\circ}(38.5 \pm 5.5 \mathrm{~cm})$. The Index of Difficulty, as defined by Fitts' Law [9], covers a large range to account for different behaviors at varying sizes. Reed et al. [10] discuss how Fitts' Law applies to two people working together. The subjects are instructed to move into the target and hold it there until a new one appears. The subjects hold the handle within the target for a random amount of time ranging from 700 to $1700 \mathrm{msec}$. The new targets appear on the opposite side as the previous trial, so the targets alternate sides of the work space. One sixth of the targets are catch trials where the new target appears in the same direction as the previous trial - catch trials do not switch sides. Catch trials, varying the timing of the wait time, and moving the target center prevented the subjects from adapting to a regular back-andforth pattern.

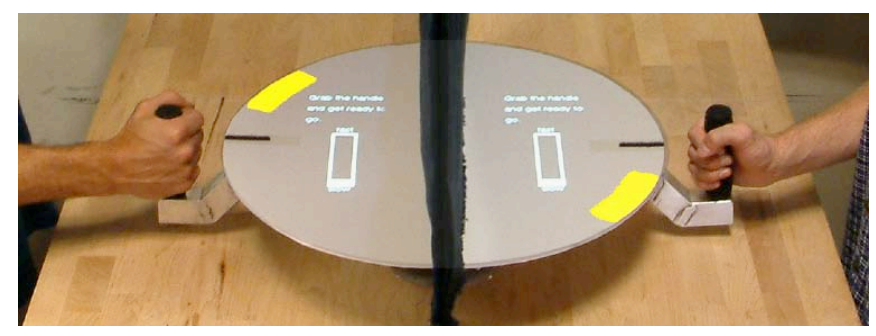

Fig. 1. Experimental setup with two-handled crank. A projector displays the targets from above. A curtain hangs between the two subjects to prevent non-physical communication.
An experimental run starts with subject A performing a set (120 targets) alone. Subject B then performs a set alone. Next, subjects A and B work together on a set of 120 targets. The whole sequence $(\mathrm{A}, \mathrm{B}, \mathrm{AB})$ is performed twice. The sequence was flipped $(\mathrm{AB}, \mathrm{A}, \mathrm{B})$ for the last four subject pairs to determine order effects. No order effects were found. For each set, subjects performed 100 alternating direction trials with 20 catch trials (discarded in data analysis). The experimental apparatus was identical when the subjects were working alone and when working together, except that the small inertia of the crank was doubled in the two-subject condition. The entire experiment took less than 30 minutes to complete a total of 720 trials (480 for each subject).

\section{ForcE ANALYSIS}

Each of the subjects' forces were measured separately using strain gauges on each handle. We defined the sum of the subjects' forces as the "net force," since it is the force that directly contributes to accelerating the crank. The net force necessarily matches the measured angular acceleration of the crank. We defined the difference of the subjects' forces as the "difference force." The difference force has no effect on the crank acceleration, thus it does not contribute directly to task completion; it is in some sense a measure of "wasted" energy. However, as will be shown in the Results, we note that the difference force is often significantly larger in magnitude than the net force, and exhibits considerable structure during the late phase of the task (500 - $900 \mathrm{msec})$. This raises the distinct possibility that the difference force reflects a meaningful channel of interaction between the subjects.

\section{A. Net Force}

The net force was found to be similar for all trials across all dyads. A characteristic trace of the net force is shown in Fig. 2. The net force is calculated by adding the two subjects' forces together during the dyad trials. The net force is very similar to the force from a single subject performing the task (not shown). The peak acceleration and deceleration forces are generally around 7 Newtons. The individual forces of the net force from each subject can take any form.

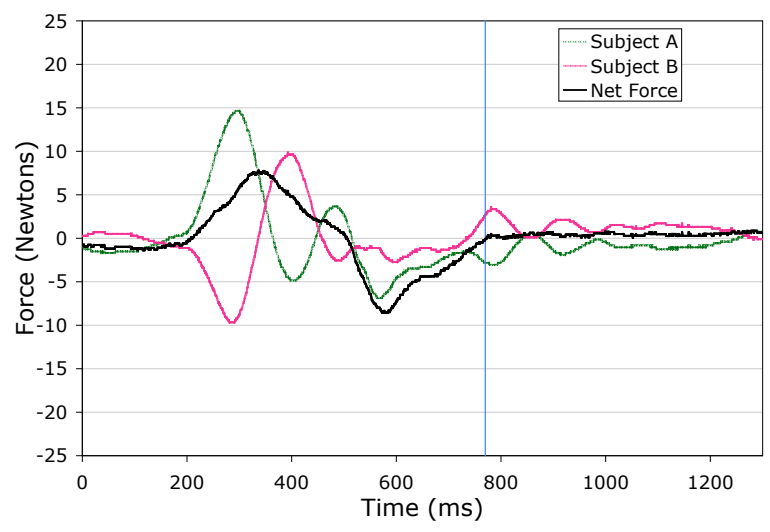

Fig. 2. Typical net force for a dyad performing the task. The net force is the sum of the two subjects forces and is the portion of the subjects' force that goes into accelerating the crank. 


\section{B. Phases of Motion}

All trials display a characteristic pattern: an initial delay, accelerating, decelerating, and final positioning. These are found numerically as defined below.

The reaction time of the dyad is defined as the time of the first motion occurrence after the target is displayed. The reaction time was generally in the range of $200-280 \mathrm{msec}$ and was slightly faster in the dyad cases compared to the faster of the two individuals.

The next phase is acceleration $(t=200 \mathrm{msec}$ to $t=500$ msec in Fig. 2). The force generally ramps up and then ramps back down into the deceleration phase $(t=500 \mathrm{msec}$ to $t=900 \mathrm{msec})$. The vertical line shows the completion time; this is when the dyad first reached and remained within the target. Once the subjects have reached the target, their net force quickly decreases to zero. In general, the deceleration phase was not found to be as uniform across trials.

\section{Difference Force}

Although the net force is similar to the force of one subject performing this task alone, each subject performing the task in a dyad will exert dissimilar forces. The difference forces can have very different structures depending on how the subjects performed the task. Two types are shown in Figs. 3 and 4 . The difference force is calculated by subtracting one subjects' force from the other during the dyad trials. The graph could be mirrored around the X-axis if the two subjects had switched places, so only the characteristic shape is relevant. Difference forces can range from 0, if they are in perfect agreement, to more than \pm 30 Newtons for an argumentative dyad.

Fig. 3 shows a typical difference force for a pair with what we might call a "lazy" subject. During the acceleration phase, the difference force is positive, which reveals that subject $\mathrm{A}$ is pushing toward the target and subject $\mathrm{B}$ is pushing

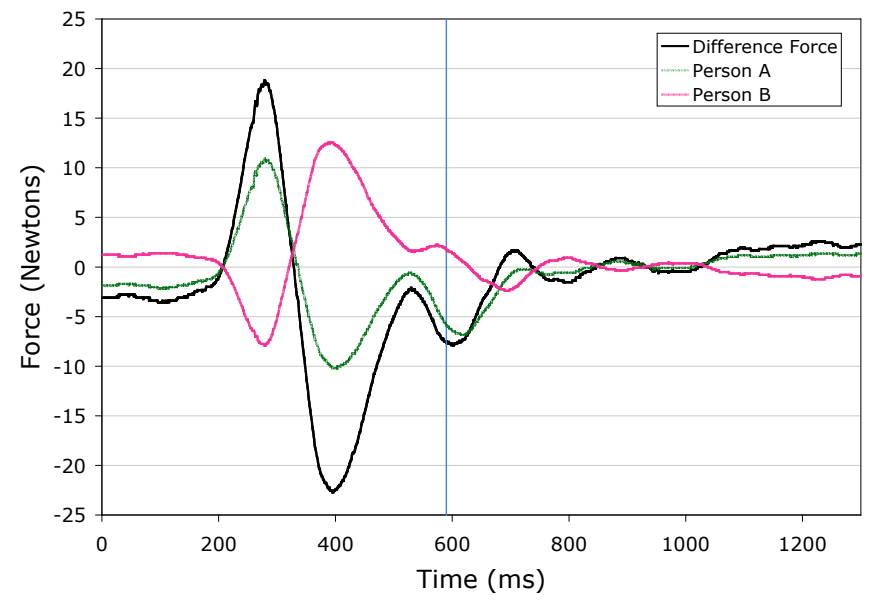

Fig. 3. The difference force is the difference between the two subjects' forces. This profile changes sign in the middle indicating that the direction each subject was pushing changed. The "dominant" subject A pushes toward the target in the beginning to accelerate the crank and away from the target in the end to decelerate the crank. The "lazy" subject B applies very little helpful force throughout the motion. away from it. As they approach the target, the difference force changes from positive to negative showing that the direction each subject is pushing has changed, so subject A is decelerating the crank. Subject A both accelerated and decelerated the crank. Subject B has opposed all the changes in velocity of subject $A$ throughout the trial. In other words, subject $B$ has acted just like a mass at the end of the handle.

A second example of a difference force profile is shown in Fig. 4, in which the difference force is always positive. In this example, the difference force does not change sign indicating that neither subject has changed the direction of their force. In the acceleration phase, subject A accelerates the crank while subject B opposes. In the deceleration phase, they switch roles; subject $\mathrm{B}$ decelerates the crank while subject A opposes. In this trial, each subject is only pushing the crank in one direction for the entire trial - they never switch the direction they are pushing, even though the crank both accelerates and decelerates. In this case, each subject specializes to only pushing one direction.

Two features are noteworthy about the difference forces shown in Fig. 3 and Fig. 4. First, in both cases, there is a considerable, and typical, oscillation after the dyad has reached the target $(t=600 \mathrm{msec})$. This oscillation appears only in the difference force, not in the net force or in the motion of the handle.

Second, neither of the force profiles start nor end at zero. Instead, dyads maintain a constant level of small difference force at the end of each trial, perhaps similar to how your biceps and triceps can work against each other to hold your hand still. Both this constant difference force and these oscillations will be discussed further in the Results.

\section{RESULTS}

Data from 28 pairs (56 subjects) is analyzed (data from one additional pair had to be discarded).

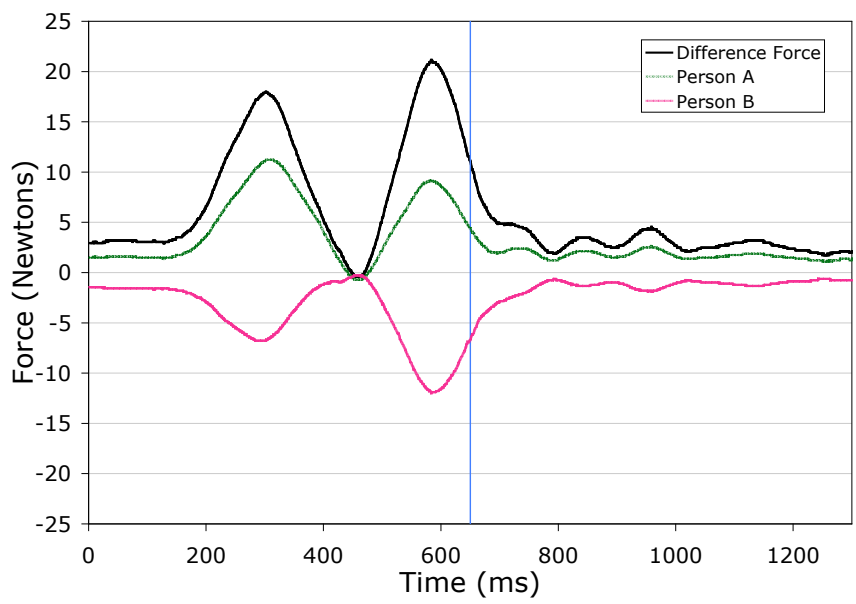

Fig. 4. A second example of a difference force profile. Here the sign of the difference force is always positive, indicating that each subject pushed the same direction (i.e. only toward the target or only away from the target) for the duration of the trial. Subject A accelerated the handle, pushing in only one direction and subject B decelerated the handle, pushing in only the opposite direction. 


\section{A. Specialization}

We know the total force contribution from each user $\left(F_{1}, F_{2}\right)$ and the net force of the subjects $\left(F_{n e t}\right)$, so the contribution (ranging from 0 to 1$)$ of each user $\left(C_{1}, C_{2}\right)$ to the overall acceleration is given by equations (1), (2), and (3).

$$
\begin{gathered}
C_{A}=\frac{F_{A}}{F_{n e t}} \\
C_{B}=\frac{F_{B}}{F_{n e t}} \\
C_{A}+C_{B}=1
\end{gathered}
$$

A dominant subject has a contribution of 1 , indicating that they were providing all of the necessary forces to cause motion and a contribution of 0 indicates they provided no beneficial force.

To determine if both subjects are performing the same or different tasks, the average contribution of each subject during the acceleration phase is plotted against the average contribution of each subject during the deceleration phase, shown in Fig. 5. Each subject is plotted, so there are 56 symbols representing the two individuals from the 28 dyads. Since the dyads are coupled and (3) holds, each subjects' partner is mirrored around the point $(0.5,0.5)$.

Points that lie along the line $x=y$ represent subjects that contribute equally in the acceleration and deceleration phases. A subject that lies at $(1,1)$ is completely dominating the task while his partner at $(0,0)$ is doing nothing to help move the crank. Points that lie along the line $x=1-y$ represent subjects that share the task between them. A subject that lies at $(0,1)$ or $(1,0)$ contributes completely in half of the

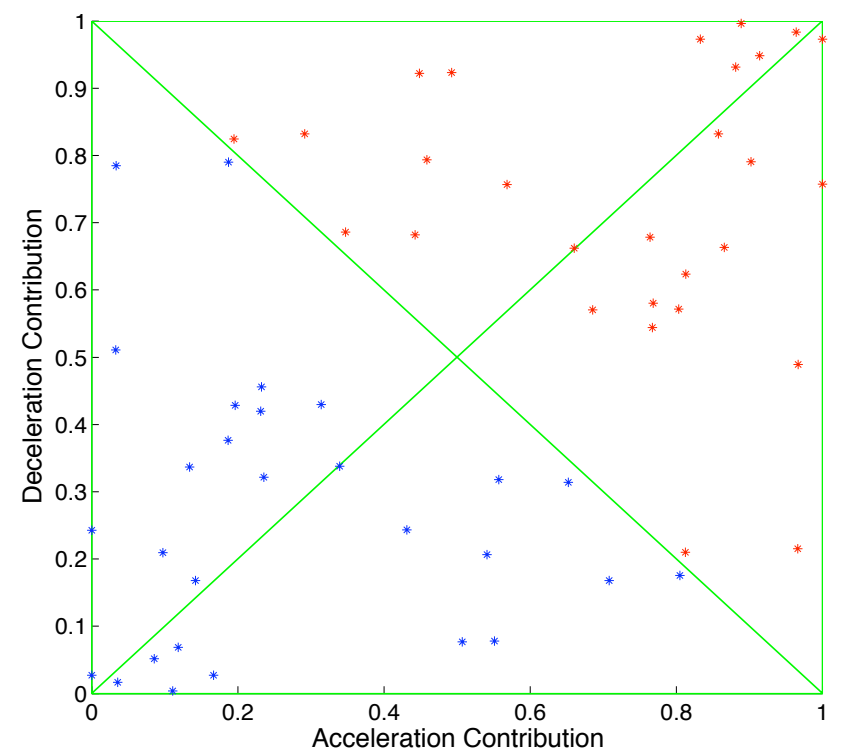

Fig. 5. Contribution percentage for accelerating and decelerating phases of the task for each subject. $x=y$ line represents equal contribution in each direction. $x=1-y$ line represents specialization since each subject contributes differently in each direction. These subjects do not necessarily lie along the equal contribution or the specialization line. The large scatter indicates that some of the dyads perform this task in a specialized way, but this is not uniform among all subject pairs. task and contributes nothing in the other half. This indicates a specialized dyad - a dyad with two subjects performing different aspects of moving the crank. Fig. 5 shows that many of the dyads lie along the specialized line, although this is not universal among all the dyads.

Alternately, Fig. 6 shows the contribution forces divided by the subjects pushing the crank to the left versus pushing the crank to the right. These points all lie along the line $x=y$ indicating that specialization does not appear on a left versus right basis. Given these two modes of specialization, many of these subjects show that they specialize in regards to accelerating/decelerating regardless of the direction they are pushing.

The subjects do not change their pattern much as the experiment progresses. They quickly fall into a regular pattern of completing the task. The standard deviation is generally less than 0.5 in each direction, but a couple dyads had standard deviations as high as 1.5 in both directions. The consistency of the dyads does not increase as the experiment continues.

\section{B. Difference Force Oscillations}

When a dyad is in the target zone, the difference force contains oscillations at a frequency in the $6-10 \mathrm{~Hz}$ range while the net force shows much smaller oscillations. This indicates that the two subjects in the dyad began to push in antiphase with each other sometime before they reached the target and continued after they are within it.

This activity in their difference force is not consistent among all subjects. Subjects that generally perform the task quicker generally have a higher power level in the $6-10 \mathrm{~Hz}$ range of their difference force. Since it is expected that faster dyads would have more high frequency activity, we have

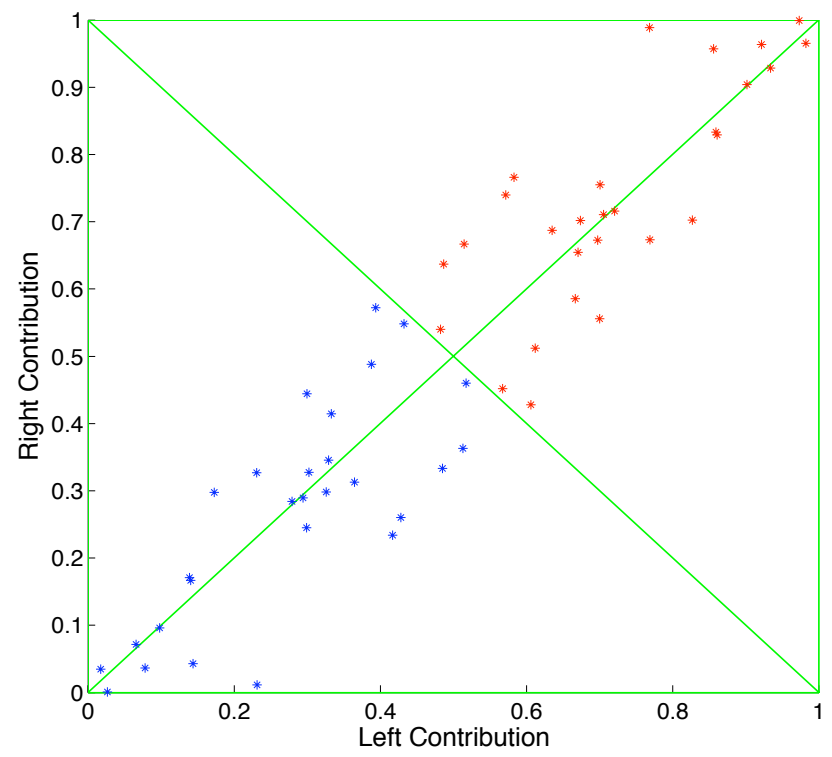

Fig. 6. Contribution percentage for pushing to the left and right for all 56 subjects. These generally lie along the equal contribution line. Points outside the box represent points where the subject is pushing harder than necessary due to additional forces from his or her partner. 
scaled the cutoff frequency proportional to their speed. The cutoff frequency ranges from $4.8 \mathrm{~Hz}$ (slow dyads) to $7.2 \mathrm{~Hz}$ (fast dyads) for the high pass frequency. The bandwidth is normalized to $4 \mathrm{~Hz}$ so the low pass frequency ranges from $8.8 \mathrm{~Hz}$ (slow dyads) to $11.2 \mathrm{~Hz}$ (fast dyads). Filtering is done with a 2 pole digital Butterworth filter. Fig. 7 shows the RMS of these bands plotted against the time to completion.

\section{Steady Dyadic Opposition Force}

At the end of each trial, the subjects bring the crank to rest as they wait for the next target to be displayed. If a subject is working alone, then there is no way to apply any force to the handle with no acceleration. If there are two subjects, each subject can apply a force even though the crank is not moving, as long as the forces are equal and opposite. This generates a difference force with no net force, hence no acceleration.

We find that the subjects exert a significant force in opposition to one another (averaging 4 Newtons). We speculate that this force serves to increase stiffness for the dyad in the same way that muscle co-contraction increases arm stiffness for an individual. Gribble [11] has shown that for individuals the extent of co-contraction correlates inversely with target size in a Fitts-like task. Our results for dyads show a correlation of opposition force with target size, but not for all dyads. Fig. 8 shows the distribution of the average dyadic cocontraction for each pair at the end of each trial. This shows a co-contraction strategy similar to those used in parallel robotics and in human bimanual control [12] [13].

\section{Discussion}

When a single person becomes part of a dyad, many new solutions to completing the task develop [14] [15]. The various types of difference force profiles show that the subjects are performing this task differently than they would alone, yet the net force profile is very similar to how they would perform it alone.

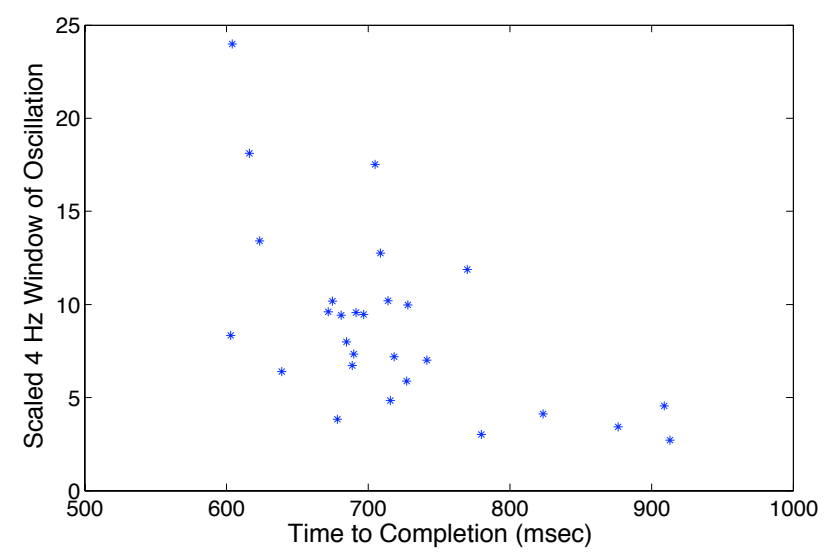

Fig. 7. The amount of oscillation in a band around $6-10 \mathrm{~Hz}$ for the difference force. It is expected that there be more high frequency activity with faster dyads, so this band has been scaled by the time to completion; faster dyads are compared to high frequencies and slower dyads are compared to lower frequencies. There is still a general trend of decreasing oscillations with decreasing completion time.

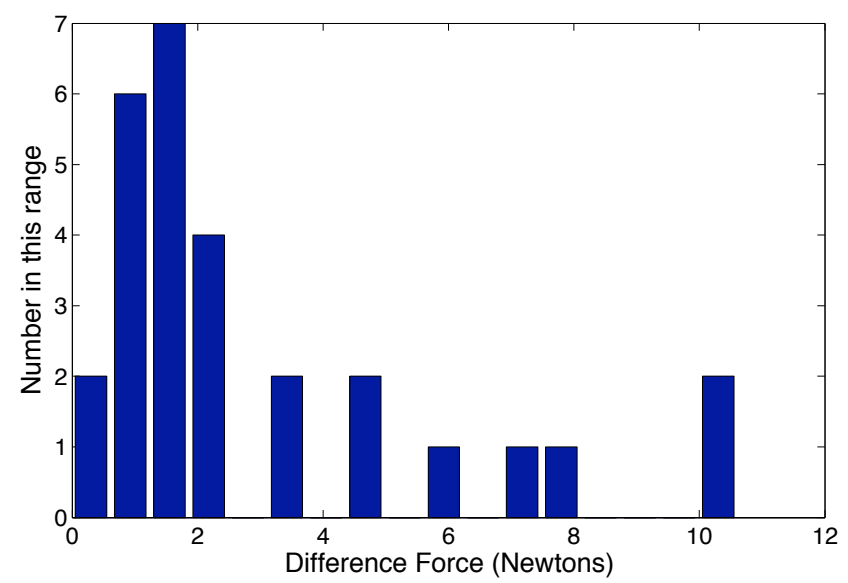

Fig. 8. The average difference force at the end of trial for all 28 dyads. Six of the dyads show significant force that they maintain while they are waiting for the next target to appear. In order to complete the task, no force is required at this point.

It seems these subjects are communicating something with each other through the crank during the experiment. While working together, some of the subjects would smile or even laugh with their partner, even though they could not see each other. Some of the subjects reported that they knew the other subject was in control and some reported that it felt more stable when working together.

\section{A. Specialization}

The subjects could tell what the other subject was doing and in some cases, the two subjects learned to work together. The learning took place very quickly at the beginning of the experiment. Scheidt et al. [16] provide evidence that with uncertain events, humans will use only the past few trials to generate optimal motor responses, so learning will happen very quickly. In these experiments, the environment is not completely uncertain, but initially working with a partner could be considered so. This would imply that subjects that are going to specialize are going to specialize quickly and stay there. Subjects may develop a comfortable, but less than ideal, cooperation, such as the specialization that developed. If we can find a better cooperation, then disrupting this mediocre cooperation should encourage all subjects to specialize more efficiently, leading to an improved method for interaction between two people.

\section{B. Difference Force Oscillations}

As shown in the representative difference force plots (Fig. 3 and Fig. 4), the subjects have become in anti-phase with each other. The crank is not moving, yet the subjects are changing their forces in sync with each other. Humans are easily able to synchronize their limbs, such as legs and arms, during walking [17], simultaneously drawing circles with both hand [18], and in other bimanual tasks [19]. It is possible that during this target acquisition task, the two subjects were learning to synchronize with each other while still maintaining the desired location. 


\section{Steady Dyadic Opposition Force}

The dyadic co-contraction shows a similar result as Shergill et al. [20] where they show that two people will naturally escalate the force applied to them. The differences between their experiment and this one is that our subjects could apply a force in two directions, not just one, and that the subjects primary task is moving a handle instead of applying the same force that was previously applied to them. After this crank experiment, several of the subjects asked if they had the same targets as their partner. They had a hard time believing the targets were identical since they were sure their partner was trying to move to a different target. This dyadic co-contraction could be a form of haptic communication (or miscommunication in this case) since the subjects are able to make an assessment of their partner based upon this force. This co-contraction force may also serve to stabilize the two person system, just like a single persons' agonist and antagonist muscles.

\section{FUTURE WORK}

\section{A. Perturb Specialization Roles}

Since these subjects seem to quickly fall into their roles in this task, we would like to perturb these roles and observe the quick adaptation process. There are multiple ways to disrupt the subjects' cooperation. We can present the target to the slow subject before the faster subject to give them a head start, presumably, this would encourage the slow subject to adopt an "accelerating" role. Alternatively, to encourage the faster subject to adopt the "decelerating" role, we could show him or her a smaller target than the other subject. Presumably, this would cause this subject to determine the fine position while the other subject only cares about the general placement within his or her larger target.

In the case of a dominant subject, we can give the less dominant subject a power advantage by actuating the motor in proportion to the measured strain gauge signal. We can add to or subtract from either subject's effort, even to the point of completely disempowering one subject.

\section{B. Measure Individual Co-contraction}

We observed dyadic co-contraction in most of the subjects as shown by their difference force. If this dyadic force can fill the role of co-contraction, then we would expect a reduced co-contraction within each subject. This could either be determined by using EMG's or by applying a small torque and measuring the response from each individual. The subjects will be applying opposing forces so each subject should be more resistant in one direction than the other.

To further study this dyadic co-contraction, we can present the subjects with overlapping but not identical target regions to explore negotiation and physical compromise. Presumably, this dyadic co-contraction force will increase since each subject would prefer to be in the center of his or her target.

\section{ACKNOWLEDGMENTS}

The authors gratefully acknowledge the advice and guidance of Peter Vishton, Marcia Grabowecky, and Satoru Suzuki, as well as ongoing discussions with Kevin Lynch.

\section{REFERENCES}

[1] Rehabilitation Institute of Chicago. Gait restoration/lokomat project. http://www.smpp.nwu.edu/mars/Project2.htm, 9/13/2004.

[2] N. Wegner and D. Zeaman. Team and individual performance on a motor learning task. Journal of General Psychology, 55:127-142, 1956.

[3] R.E. Shaw, E. Kadar, M. Sim, and D.W. Repperger. The intentional spring: A strategy for modeling systems that learn to perform intentional acts. Journal of Motivational Behavior, 24(1):3-28.

[4] E. Sallnas and S. Zhai. Collaboration meets Fitts' law: Passing virtual objects with and without haptic force feedback. Proceedings of INTERACT, IFIP Conference on Human-Computer Interaction, pages 97-104, 2003.

[5] I. Elhajj, H. Hummert, N. Xi, and Y. Liu. Real-time haptic feedback in internet-based telerobotic operation. IEEE International Conference on Robotics and Automation, San Francisco, 2000.

[6] J. P. Hespanha, M. McLaughlin, G. S. Sukhatme, M. Akbarian, R. Garg, and W. Zhu. Haptic collaboration over the internet. Fifth Phantom Users Group Workshop, Aspen, CO, 2000.

[7] David P. Barnes and Michael S. Counsell. Haptic communication for remote mobile manipulator robot operations. In American Nuclear Society, Proc. 8th Topical Meeting on Robotics and Remote Systems, 1999.

[8] Paul Griffiths and R. Brent Gillespie. Shared control between human and machine: Haptic display of automation during manual control of vehicle heading. Haptics 2004, pages 358-366, 2004.

[9] P. M. Fitts. The information capacity of the human motor system in controlling the amplitude of movement. Journal of Experimental Psychology, 47:381-391, 1954

[10] Kyle Reed, Michael Peshkin, J. Edward Colgate, and James Patton. Initial studies in human-robot-human interaction: Fitts' law for two people. International Conference of Robotics and Automation, 2004.

[11] P.L. Gribble, L.I. Mullin, N. Cothros, and A. Mattar. Role of cocontraction in arm movement accuracy. Journal of Neurophysiology, 89:23962405, 2003

[12] J. L. Patton and P. Elkins. Training with a bimanual-grasp beneficially influences single limb performance. Society for Neuroscience, Orlando, FL, USA, 2002.

[13] V.S. Chib, J.L. Patton, K.M. Lynch, and F.A. Mussa-Ivaldi, . Haptic discrimination of perturbing fields and object boundaries. HAPTICS 2004, 0-7695-2112-6/04, 2004.

[14] A. Karniel, R. Meir, and G. F. Inbar. Exploiting the virtue of redundancy. International Joint Conference on Neural Networks, Washington, DC, 1999.

[15] F. Lacquaniti and C. Maioli. Distributed control of limb position and force. Requin GESaJ (ed) Tutorials in Motor Behavior II, pages 3154, 1992.

[16] Robert A. Scheidt, Jonathan B. Dingwell, and Ferdinando A. MussaIvaldi. Learning to move amid uncertainty. Journal of Neurophysiol, 86, 2001.

[17] T. Wannier, C. Bastiaanse, G. Colombo, and V. Dietz. Arm to leg coordination in humans during walking, creeping and swimming activities. Experimental Brain Research, 141(3):375-379, 2001.

[18] R. J. Carson, J. Thomas, J. J. Summers, M. R. Walters, and A. Semjen. The dynamics of bimanual circle drawing. The Quarterly Journal of Experimental Psychology: Section A, 50(3):664-683, 1997.

[19] C. M. B. Evans and S. N. Baker. Task-dependent intermanual coupling of $8 \mathrm{-hz}$ discontinuities during slow finger movements. European Journal of Neuroscience, 18:453-456, 2003.

[20] S. S. Shergill, P. M. Bays, C. D. Frith, and D. M. Wolpert. Two eyes for an eye: The neuroscience of force escalation. Science Magazine, 301:187, 2003. 